\title{
Analysis of hot spot directional signatures measured from space
}

\author{
Francois-Marie Bréon and Fabienne Maignan \\ Laboratoire des Sciences du Climat et de l'Environnement, Commissariat à l'Energie Atomique, Gif sur Yvette, France
}

Marc Leroy

Centre d'Etudes Spatiales de la Biosphère, Toulouse, France

Ian Grant

CSIRO Atmospheric Research, Aspendale, Australia

Received 16 July 2001; revised 15 October 2001; accepted 17 December 2001; published 22 August 2002.

[1] Reflectance measurements from the spaceborne Polarization and Directionality of Earth Reflectances (POLDER) instrument are used to analyze the so-called hot spot directional signature in the backscattering direction. The hot spot is measured with an angular resolution better than half a degree using the directional capabilities of the radiometer, with some assumptions on the spatial homogeneity of the surface. The analysis yields the first quantitative observation of the hot spot signature of vegetated surfaces, with such angular resolution. The measurements show that the hot spot reflectance is a function of the phase angle $\xi$ rather than a function of a parameter $\Delta$, often used in hot spot modeling, that quantifies the horizontal distance between Sun and view directions. The observed directional signature is very accurately fitted by a linear ratio of the phase angle, as predicted by a simple theory of radiative transfer within the canopy foliage. Most of the measured hot spot half widths are between $1^{\circ}$ and $2^{\circ}$. Some dispersion occurs for the cases belonging to the forest and desert International Geosphere-Biosphere Program (IGBP) classes, in the range $1^{\circ}$ to $5^{\circ}$. Theory predicts that the width is independent of wavelength. Our measurements indicate that the widths at 670 and $865 \mathrm{~nm}$ are very close, but with a significant scatter in regards to the rather small variability. The distribution of the width as a function of the IGBP surface classification shows a variability within the classes that is larger than between the classes, except for the "evergreen broadleaf" class. The hot spot reflectance amplitude is generally on the order of $0.10-0.20$ at $865 \mathrm{~nm}$ and $0.03-0.18$ at $670 \mathrm{~nm}$, although the full range of values is wider. For thick canopies, it may be interpreted in terms of foliage element (leaf) reflectance. Retrieved values are on the order of 0.4 in the near infrared and in the range $0.05-0.20$ at $670 \mathrm{~nm}$. At $440 \mathrm{~nm}$, the amplitude of the signature is very small, as is expected from the small surface reflectance. This confirms that the atmospheric contribution to the reflectance increase at the backscattering direction is negligible. INDEX TERMS: 0659 Electromagnetics: Random media and rough surfaces; 0649 Electromagnetics: Optics; 0933 Exploration Geophysics: Remote sensing; KEYWORDS: hot spot, POLDER, backscattering

\section{Introduction}

[2] The so-called hot spot, or opposition effect, refers to the peak of reflectance obtained when Sun and view directions coincide. This effect has been known for over a century [Seeliger, 1895] and has been observed over a variety of bodies, including the Moon, Mars, asteroids, planetary satellites, and terrestrial vegetation. The widely accepted explanation for the hot spot effect is shadow hiding, in which particles at the surface (leaves, soil grains) cast shadows on adjacent particles. Those shadows are visible at large phase angles (the angle between the Sun

Copyright 2002 by the American Geophysical Union. 0148-0227/02/2001JD001094 and view directions), but at zero phase angle they are hidden by the particles that cast them. Coherent backscatter is another physical cause of enhancement of reflectance in the hot spot direction [Kuga and Ishimaru, 1984; Hapke et al., 1993]. It is based on the fact that portions of wave fronts that are multiply scattered within a nonuniform medium and follow the same path, but in opposite directions, combine constructively at zero phase angle. Laboratory experiments suggest that shadow hiding dominates the hot spot for most terrestrial surfaces [Woessner and Hapke, 1987; Hapke et al., 1996].

[3] The study of the hot spot is of interest for the remote sensing community for several reasons. The width of the peak in the shadow-hiding model depends on the ratio of the 
interparticle spacing to the extinction distance, which could be a discriminating structural parameter of vegetation canopies [Gerstl and Simmer, 1986]. The observation in the exact backscattering direction, where no shadow is visible, gives access to the reflectance of foliage elements or soil grains. This in turn should lead to the leaf chemical properties such as chlorophyll concentration [Jacquemoud and Baret, 1990]. It should be noted also that the hot spot width is expected to be relatively invariant to atmospheric perturbations, such as aerosol loading [Powers and Gerstl, 1988]. Several shadow-hiding models have been elaborated, in the context of studies of planetary regolith [Hapke, 1986] or terrestrial vegetation [Kuusk, 1985, 1995; Jupp and Strahler, 1991; Chen and Leblanc, 1997].

[4] Despite their interest, observations of the hot spot on vegetation canopies are scarce. Ground measurements of this effect are generally perturbed by the shadow of the sensor, except if the sensor is appreciably elevated as in the work of Myneni and Kanemasu [1988]. Several hot spotmeasurements have been obtained from a helicopter [Bréon et al., 1997]. Because most spaceborne optical sensors acquire crosstrack measurements, they do not sample the principal plane and are not well suited to observing the hot spot signature.

[5] The directional capabilities of the spaceborne Polarization and Directionality of Earth Reflectances (POLDER) instrument [Deschamps et al., 1994] offer an opportunity to sample the surface BRDF (bidirectional reflectance distribution function) including its hot spot component. The instrumental principle is roughly that of a standard wide field-of-view camera. The displacements of the camera (along track, or from different orbits) are such that a point on Earth is observed under different directions. Thus one obtains the BRDF of every pixel of the globe at the resolution of $6 \mathrm{~km}$ in a few days, providing that atmospheric conditions are favorable [Hautecoeur and Leroy, 1998; Bicheron and Leroy, 2000]. However, the angular resolution of the BRDF measurements, limited by the amplitude of the displacements between two acquisitions is on the order of $6^{\circ}\left(9^{\circ}\right.$ at nadir). This is not sufficient to determine the shape of the hot spot.

[6] In this paper we measure the hot spot with POLDER data using a different measurement principle. Close to the backscattering direction, the apparent spatial variability of the reflectance is generated by the directional signature. The analysis of targets of opportunity with such geometry yields a measure of the hot spot reflectance signature with very fine angular resolution, but limited to a small number of sufficiently homogeneous areas. Grant et al. [2002] have shown that this principle is operative with a few POLDER images taken on an Australian site.

[7] We perform a systematic exploration of the hot spot signatures using this principle with the eight months of available POLDER data over the whole globe. Several thousand hot spot signatures have been collected. We have kept the best quality signatures to make a statistical analysis of the main characteristics of the hot spot feature, that is, its amplitude and width.

\section{The POLDER Measurement Principle}

[8] POLDER was launched onboard the Advanced Earth Observing Satellite (ADEOS) in August 1996. Acquisition was quasi-continuous from late October to the end of June of 1997, when the failure of the platform solar panel terminated the operation of all instruments on board. Nevertheless, eight months of measurements are available and allow original studies thanks to the uniqueness of the measurement principle. Another similar instrument is scheduled for launch onboard the ADEOS-II platform in February 2002. The instrument is composed of a wide field-of-view lens, a filter wheel and a detector. The filter wheel permits radiance measurements in eight spectral bands from $440 \mathrm{~nm}$ (blue) to $910 \mathrm{~nm}$ (near IR, water vapor absorption). The detector is a bi-dimensional CCD array with $242 \times 274$ independent sensitive areas. One snapshot yields an image of a portion of the Earth of size roughly $2400 \times 1800 \mathrm{~km}^{2}$, similar to what a camera with a wide field-of-view lens would provide, with a spatial resolution on the order of $6 \mathrm{~km}$. The pixels in the image are viewed with various zenith angles and azimuths. The zenith angle at the surface varies between $0^{\circ}$ at the image center, to $60^{\circ}$ crosstrack and $50^{\circ}$ forward and aft.

[9] One such snapshot is acquired, for each spectral band, every $20 \mathrm{~s}$. There is a large overlap of the areas observed by successive snapshots. Thus a surface target is observed from varying directions as the satellite goes along its orbit. The reflectance of a target in the instrument swath will be acquired between 10 and 14 times depending on its position with respect to the satellite subtrack.

[10] In most cases, depending on the solar position with respect to the satellite, there is one pixel that is observed exactly (at the POLDER pixel angular resolution) in the backscattering geometry. This pixel is where the shadow of the satellite would be seen if it were much larger. The pixels surrounding this particular pixel are observed with a slightly different viewing geometry (Figure 1). Assuming that the surface does not change significantly between the pixels (details below), this opens the way for a measurement of the reflectance directional signature within a few degrees of the backscattering direction. The variation in phase angle from the central pixel is typically $0.3^{\circ}$ per pixel. It results mostly from the change in viewing geometry rather than the change in illumination geometry.

[11] As said before, the area of interest, which is observed with a geometry close to backscattering, is also observed within a few minutes from very different directions (see Figure 1). This allows an estimate of the reflectance directional signature for larger variations of the phase angle, and also a measurement of the reflectance spatial variability when the effects of the directional signature are expected to be small. Thus one may verify that the reflectance variations observed close to the backscattering geometry are the result of the directional signature (hot spot effect), and not a spurious consequence of the surface heterogeneity.

\section{Data Processing}

[12] We have processed the full set of spaceborne POLDER measurements in three spectral bands: $440 \mathrm{~nm}, 670 \mathrm{~nm}$, and $865 \mathrm{~nm}$. These bands are little affected by atmospheric absorption. The blue $(440 \mathrm{~nm})$ band maximizes the atmospheric scattering contribution and may be used to identify a possible aerosol contribution to the hot spot signature. The surface contribution dominates in the other two bands with reflectances that depend on the surface type and the photo- 


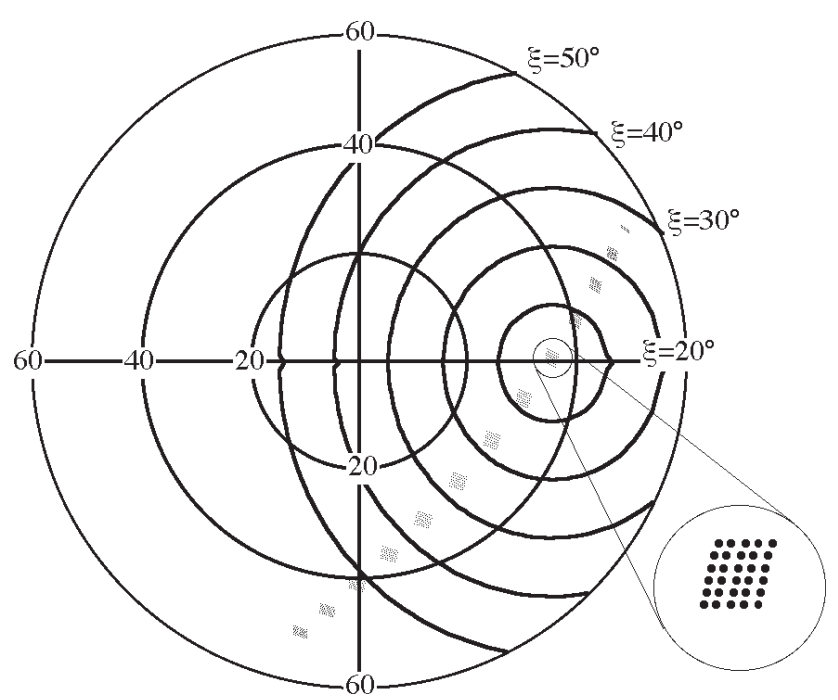

Figure 1. Typical viewing geometry for a set of $7 \times 7$ POLDER pixels used to study the hot spot directional signature. The horizontal line is the principal plane (that contains the Sun direction and local nadir). The solid circles indicate the view zenith angle by steps of $20^{\circ}$. The ellipses show the phase angle by steps of $10^{\circ}$. The 13 clusters of dots correspond to 13 successive acquisitions within roughly 2 min. Each cluster is composed of $7 \times 7$ dots corresponding to 49 contiguous surface pixels of size $(6.2 \mathrm{~km})^{2}$.

synthetic activity. For each sequence of acquisition (one sequence every $20 \mathrm{~s}$ ), we identified the surface pixel that is observed in the backscattering direction. This pixel is also observed from a number of other sequences/directions and we identified the one closest to nadir viewing. A simple test on the reflectance in the blue channel was used to eliminate cloud contaminated pixels [Bréon and Colzy, 1999]. Water covered pixels were also discarded, based on the spectral signature of the reflectance at 670 and $865 \mathrm{~nm}$.

[13] The surrounding $7 \times 7$ pixels were then extracted and the standard deviation of the reflectances was computed using the sequence closest to nadir viewing. A 0.015 threshold on the standard deviation of the reflectances at $670 \mathrm{~nm}$ eliminates heterogeneous surfaces. Within the selected area, we make a simple correction for the spatial heterogeneity using the multidirectional capabilities of POLDER: using the measurements acquired far from the backscattering direction, we compute the deviation from the spatial mean of the pixel reflectance. The bias is then subtracted from the measurements close to the hot spot. As a result of this preprocessing, several thousand valid hot spot signatures are available for analysis from the eight months of POLDER data.

[14] A simple correction for molecular scattering is also applied to the measurements:

$$
R_{\text {cor }}=\left(R_{\text {meas }}-R_{\text {mol }}\right) \exp \left[\tau_{m o l}\left(\frac{1}{\mu_{s}}+\frac{1}{\mu_{v}}\right)\right],
$$

where $R_{\text {meas }}$ is the measured reflectance, $R_{\text {cor }}$ is the corrected reflectance, $R_{m o l}$ is the molecular reflectance estimated from multiple scattering simulations [Vermote et al., 1997], and $\tau_{m o l}$ is the molecular optical thickness.
We are aware that some atmospheric effects are not accounted for in this equation, such as the aerosol contribution or the coupling between the surface reflectance and the atmospheric diffusion. However, we estimate that these are second order terms with smooth angular variations that can be neglected here since we are mostly interested in the rapid angular variations of the reflectances. The hypothesis that atmospheric scattering does not generate hot spot like signatures is verified below in the discussion section.

\section{Observed Signatures}

[15] Figure 2 shows a few examples of such hot spot directional signatures. The measurements are shown as a function of the phase angle for $7 \times 7$ pixels $\left(43 \times 43 \mathrm{~km}^{2}\right)$ centered on the exact backscattering pixel. Figure 1 shows how POLDER acquisition provides measurements on two sides of the exact backscattering direction. In order to differentiate these two groups of reflectance measurements, which are acquired with very different viewing geometries, we have assigned a negative (positive) sign to the phase angle when the corrected view angle $\theta_{v} \cos \varphi$ is smaller (larger) than the solar zenith angle $\left(\theta_{v}\right.$ and $\varphi$ are the view zenith angle and relative azimuth).

[16] Figure 2, together with a large number of similar plots derived from POLDER measurements, demonstrates that the reflectance directional signature may be derived from the apparent spatial variation even at the crude spatial resolution of the POLDER instrument. The phase angle appears to be a good classifier of the directional signature close to the backscattering direction. The purity of the directional signatures, close to the hot spot, is striking.

[17] We have selected one target for each of the major biomes according to the 17-class International GeosphereBiosphere Program (IGBP) classification [Loveland and Belward, 1997]. The "Wetlands", "Urban", "Snow and Ice" and "Ocean" classes were excluded because no suitable sites were found or, for the two latter classes, because preprocessing excluded such surfaces. Two targets were selected for evergreen broadleaf (Figures $2 b$ and 2c) and three for the desert (Figures 2n, 2o, 2p) because our global analysis showed significantly variable signatures for these classes.

[18] On each plot, the three bands are displayed. The wavelength is 440,670 and $865 \mathrm{~nm}$ from bottom to top. In some cases (Figures 2b and 2c) the blue (440) and red (670) bands are superposed. The observed signatures show several similarities. The reflectance increases almost linearly with the signed phase angle between $-40^{\circ}$ and $-10^{\circ}$. There is a much sharper increase in the very few degrees around the exact backscattering direction. The amplitude of the sharp increase appears to scale with the average reflectance. Very roughly, it amounts to between half and one third of the reflectance outside of the peak. In most cases, the sharp increase of the reflectance appears to have little impact on all POLDER sequences that are not centered on the backscattering direction. The half width of the sharp increase is most often on the order of one to two degrees, and can reach at times five to six degrees.

[19] The signature of two desert cases (Figures 2o and $2 p)$ clearly differs from the other ones. The amplitude of the 

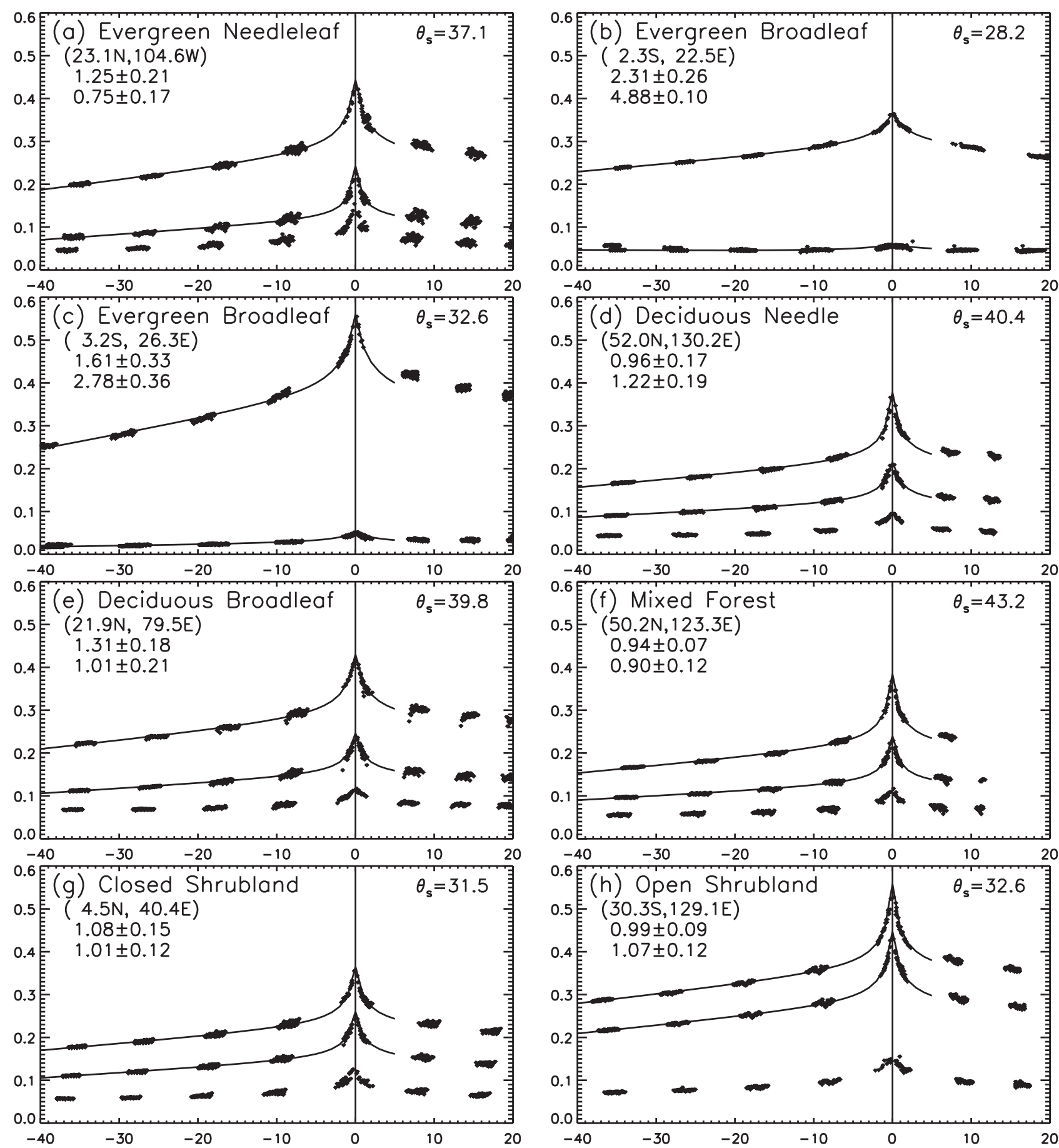

Figure 2. Hot spot directional signature as observed from the POLDER spaceborne instrument. The measured reflectance is shown as a function of phase angle. A minus sign has been applied to the phase angle when $\theta_{v} \cos \varphi<\theta_{s}$. The wavelength is $440,670 \mathrm{~nm}$ and $865 \mathrm{~nm}$ from bottom to top. The line (only for 865 and $670 \mathrm{~nm}$ ) shows the result of a best fit through the data points using the nonlinear function of equation (2). The text within the figures indicates the target type according to IGBP classification (top left) and the solar zenith angle (top right). The numbers indicate the retrieved hot spot half width for both $865 \mathrm{~nm}$ and $670 \mathrm{~nm}$ together with its uncertainty.

sharp increase is very small on these plots. Note also that the red and near infrared reflectances are very similar, consistent with typical bare soil spectral signatures. On the other hand, Figure $2 n$ indicates similar reflectances in the bands, indicating little photosynthetic activity at this desert target, but nevertheless with a large reflectance increase at backscattering. At the other extreme, the two Evergreen broadleaf cases show a much larger reflectance in the near infrared than in the two visible bands, indicating intense photosynthetic activity. The reflectances at 440 and $670 \mathrm{~nm}$ are so small that little reflectance increase is apparent close to backscattering. On the other hand, the 

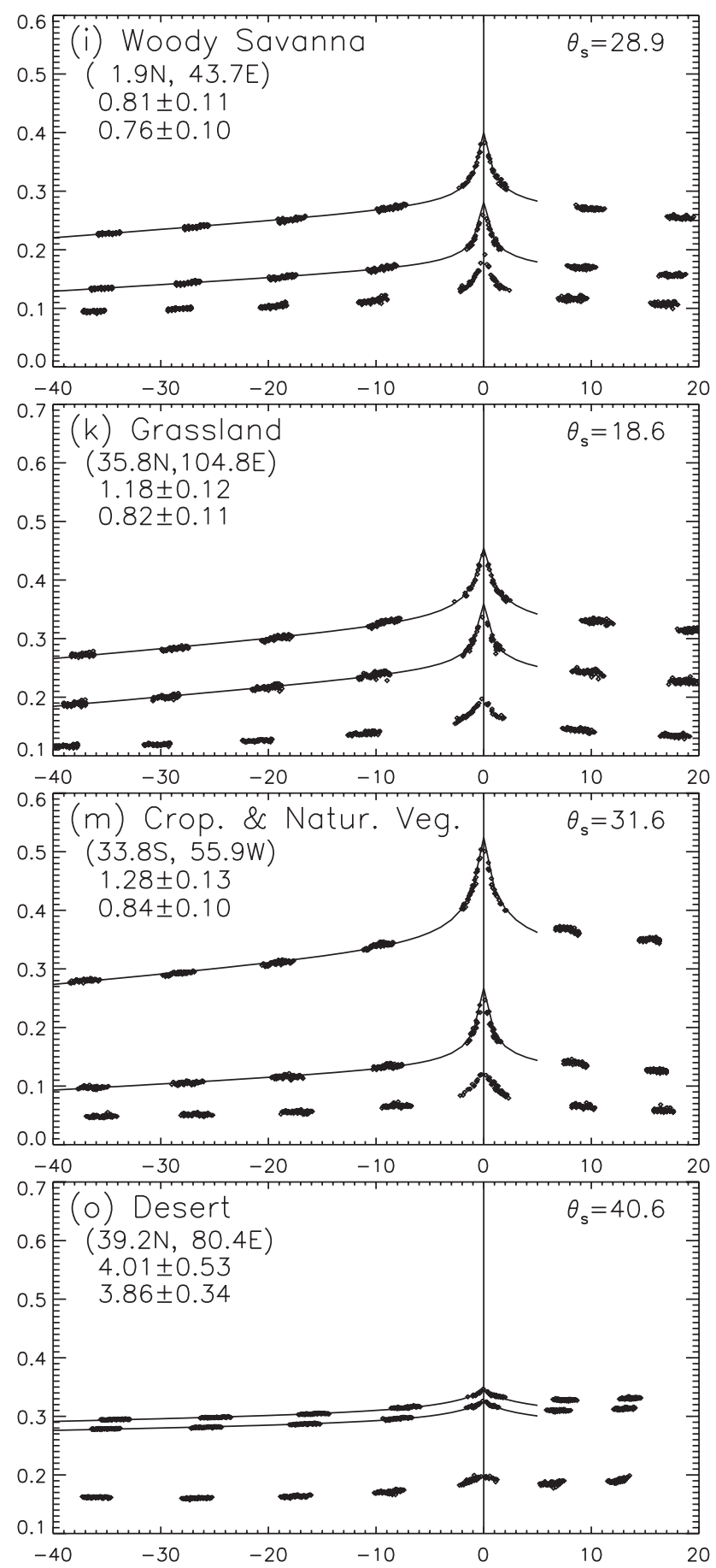

Figure 2.

near infrared signature in Figure $2 b$ shows a rather high reflectance but with a much broader reflectance peak than for other vegetated targets.

[20] Figure 2 shows that the reflectance directional signature can be fitted using a simple function of the phase angle $\xi$. The function is the combination of a linear term and a function that decreases rapidly as $\xi$ increases. For the latter, we make use of a simple linear ratio that is further justified in the theoretical section below.

$$
R_{m}=\frac{A}{1+\xi / \xi_{0}}+B+C \xi
$$
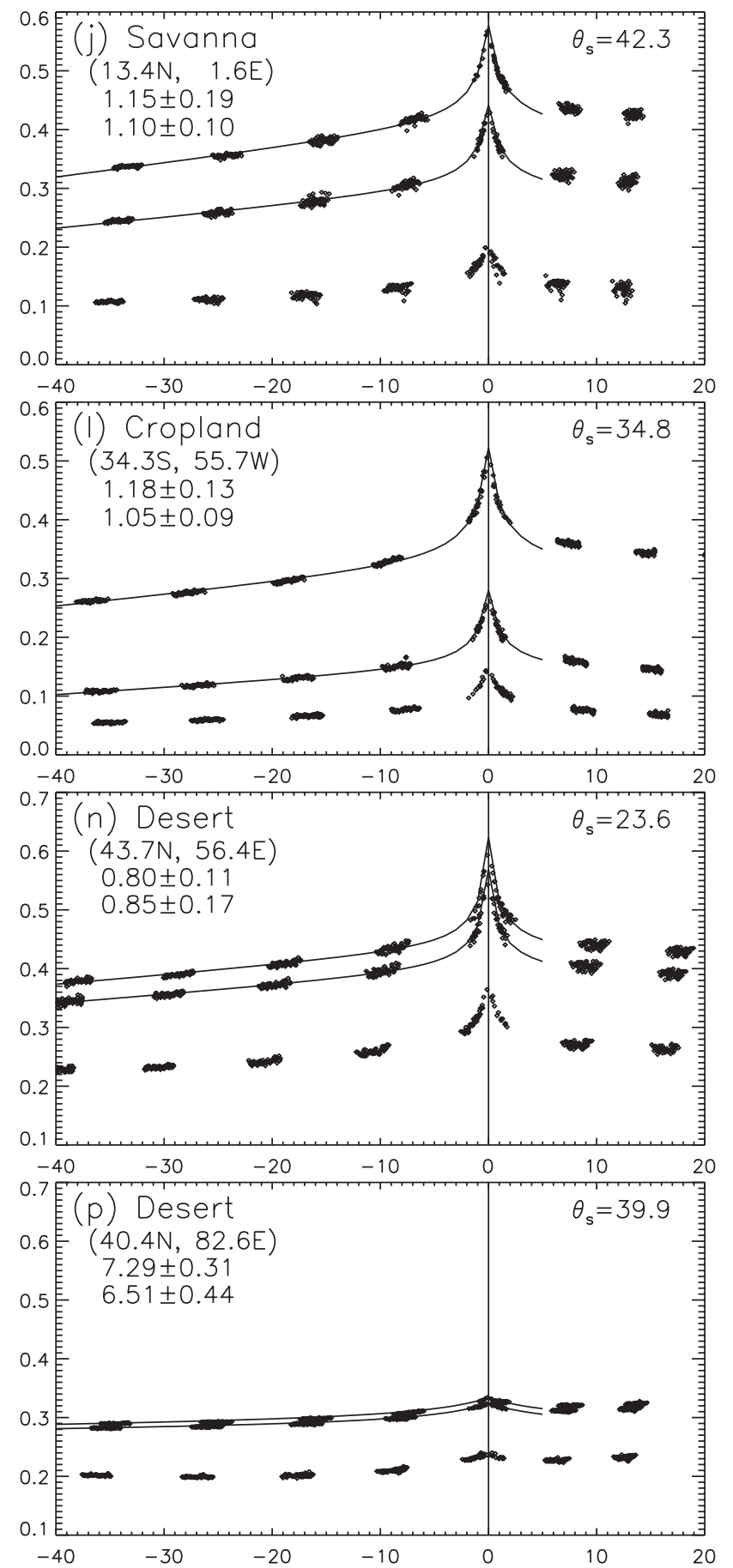

(continued)

with parameters $A, \xi_{0}, B$ and $C$. The nonlinear fit is made over measurements acquired with a signed phase angle between $-50^{\circ}$ and $+4^{\circ}$. We do not make use of the data points on the right side of the plots in Figure 2 because these are acquired with a larger viewing angle than those of the left side (for a given phase angle), and are therefore more affected by atmospheric scattering and absorption. The empirical fit yields the four parameters, together with RMS errors (RMSE) around the fit. Since this paper deals mostly with the reflectance increase within a few degrees of backscattering, we consider for the RMSE calculation only the measurements within $4^{\circ}$ of the backscattering direction. 
The RMSE is denoted $\sigma_{H S}(H S$ for Hot Spot) and the signalto-noise ratio is estimated as $\mathrm{A} / \sigma_{\mathrm{HS}}$.

[21] In order to evaluate the uncertainty on the retrieved widths, we did a Monte Carlo experiment from the data such as those shown in Figure 2. For a given hot spot signature, we randomly selected half of the measurements in the valid range of phase angle. The fit procedure yields a width estimate. We derived the uncertainty as the standard deviation of the results obtained from 100 such random samplings and fits. The numbers on the left side of Figure 2 give the hot spot halfwidth $\xi_{0}$ together with its uncertainty both for 865 (top) and $670 \mathrm{~nm}$ (bottom). The uncertainties are generally on the order of a few tenths of a degree.

[22] The hot spot phenomenon is generally interpreted as a result of correlated probabilities of transmission in the incoming and reflected paths within the canopy and soil. On the other hand, a rough surface may also generate a reflectance maximum in the backscattering direction, even if the sun rays do not penetrate it. For instance, an horizontal surface with a few vertical walls produces a broad reflectance increase toward the backscattering direction [Roujean et al., 1992]. The effect of the tree-scale structure of a canopy on the reflectance is similar to the roughness effect. Its physics is rather different from that of the correlated transmissions within the canopy, and may involve multiple scattering. The geometries involved in the two processes have similar vertical scales (the canopy depth), when the horizontal scales are very different (leaf size versus intercrown distance). The directional signature characteristic angle is related to the ratio of horizontal and vertical scales [Chen and Leblanc, 1997]. Thus we suggest that the directional signature observed within a few degrees is the result of correlated transmission, and that the broader shape, over tens of degrees, is generated by the roughness of the canopy. In the following, we focus on the directional signature for the smallest phase angles, which we refer to as the "hot spot". This is of course an idealistic view. For example, in a forest stand, the shape of the hot spot is determined by different levels of canopy structural hierarchy [Chen and Leblanc, 1997]: tree distribution pattern, crown size and shape, branches, shoots and leaves, each of these elements contributing to the hot spot feature with various characteristic widths, from $\sim 0.05^{\circ}$ for needles to several degrees for the tree structure.

\section{Theoretical Modeling}

[23] In this section, we revisit the theory of the formation of the hot spot peak by small scale elements such as leaves distributed in an homogeneous medium, in order to serve as a guide for further data reduction and analysis. We relate some canopy parameters to the reflectance directional signature observed within a few degrees from the backscattering direction. The approach followed is basically that of Jupp and Strahler [1991] of calculation of the intersection of viewed and sunlit leaf areas, but applied to the case of a canopy cover with uniform leaf orientation instead of horizontal leaves.

[24] Assuming a randomly distributed ensemble of leaves, the transmission between the top of the canopy $(\mathrm{z}=0)$ and level $z$ is

$$
T(z, \theta)=\exp \left(\frac{-1}{\cos (\theta)} \int_{0}^{z} G(t, \theta) u_{L}(t) d t\right)
$$

where $u_{L}$ is the leaf area density $\left[\mathrm{m}^{-1}\right], \theta$ is the zenith angle (either solar or view) and $G(\theta)$ expresses the mean projection of the leaf surfaces along a direction defined by $\theta$. $G(\theta)$ is $1 / 2$ for isotropically oriented leaves, as we will now assume. If the upwelling and downwelling paths were uncorrelated, the double path transmission would simply be equal to the product of the downward and upward transmissions:

$$
T^{\Downarrow \Uparrow}\left(z, \theta_{s}, \theta_{v}\right)=\exp \left[\frac{-1}{2}\left(\frac{1}{\mu_{s}}+\frac{1}{\mu_{v}}\right) \int_{0}^{z} u_{L}(t) d t\right],
$$

where $\mu_{s}=\cos \left(\theta_{s}\right)$ is the cosine of the solar zenith angle and $\mu_{v}=\cos \left(\theta_{v}\right)$ is the cosine of the view zenith angle. However, the upwelling and downwelling paths intersecting at level $z$ in the canopy are separated by a distance $d$ at level $t$. The apparent positions of the leaves at this level, with respect to the upwelling and downwelling paths, are correlated. Therefore the leaf area to consider in the integrand of equation (4) is not $u_{L}(t) \delta t$, but rather $u_{L}(t)[1-S(d / D) / 2] \delta t$, where $D$ is a typical scale of the leaves and $S$ is an overlap function which expresses the mean overlap of a leaf projected surface over itself after a shift of $d$. (See Jupp and Strahler [1991] for a rigorous mathematical demonstration.)

[25] In the simple case of horizontal circular leaves of diameter $D, S(x)$ can be expressed analytically:

$$
\begin{array}{ll}
x \geq 1 & S(x)=0 \\
x \leq 1 & S(x)=(u-\sin u) / \pi \quad ; \quad \cos (u / 2)=x .
\end{array}
$$

[26] For a random distribution of leaves of ellipsoidal shape, we have not been able to derive an analytical expression for S. However, numerical integration is rather straightforward and the results of such integration are shown in Figure 3. For circular leaves, the isotropic distribution yields a smaller overlap function than for a planar distribution. In the former case, the projection of the leaf along the line of sight is an ellipse whose overlap nulls out rapidly when the position shift is along the small axis. For the planar distribution, there is no such rapid decrease and the overlap function is then larger. In Figure 3, we have set the typical scale of leaves from their surface area. This is why there is some overlap $(S>0)$ for ellipsoidal leaves for normalized distances greater than 1 . It can be readily shown that $S(x)$ nulls out for a normalized distance equal to $r^{1 / 2}$, where $\mathrm{r}$ is the ratio between the long and the short axis. For short distances, the overlap function decreases more rapidly for ellipsoidal leaves than for circular leaves. The same interpretation as above can be given. The derivative of $S$ close to the origin is on the order of -2 with an exact value that depends slightly on the leaf shape. For circular leaves that are isotropically oriented, $S$ can be fitted with an excellent accuracy by the polynomial function $S(x \leq 1)=1-2 x+x^{2}$, and $S(x \geq 1)=0$. RMS difference between $S$ and its analytical approximation is $3 \times 10^{-3}$. 


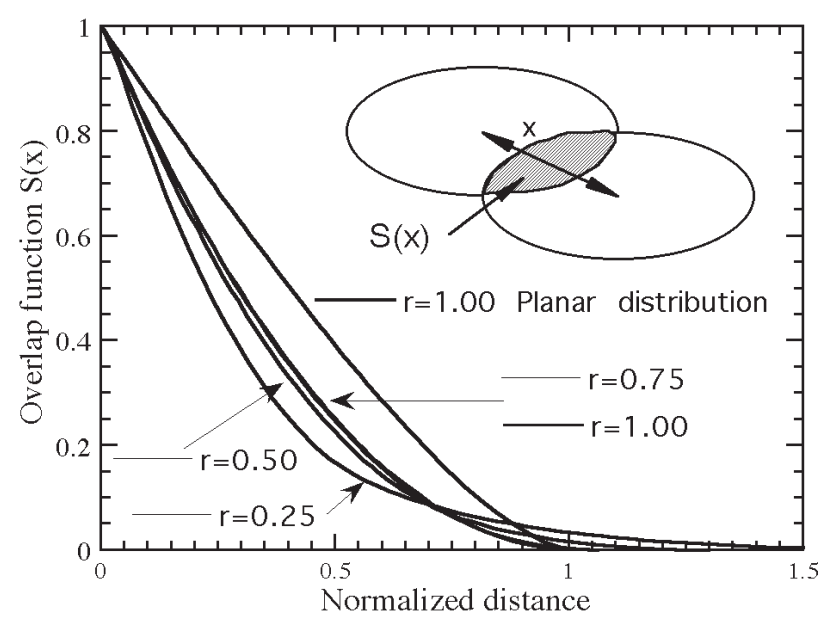

Figure 3. Overlap function $S(x=d / D)$ for various leaf shapes and orientation distributions. Leaves are assumed to be ellipsoidal with a ratio $r$ between the long and short axis. The orientation of the leaf is either planar (extreme planophile case, only the $r=1.00$ case is shown) or isotropic (all other cases). We define the typical scale of the leaves as $D=2 \sqrt{A / \pi}$ where $\mathrm{A}$ is the leaf surface area. $D$ is the diameter of the leaf for a circular shape $(r=1)$. The case $\mathrm{r}=1$ isotropic can be fitted with an excellent accuracy by the polynomial function $S(x)=1-2 x+x^{2}$.

[27] In most papers that deal with hot spot modeling [e.g., Kuusk, 1985; Nilson and Kuusk, 1989; Verstraete et al., 1990; Jupp and Strahler, 1991; Qin, 1993], the distance $d$ is equal to $(z-t) \Delta\left(\theta_{s}, \theta_{v}, \varphi\right)$ where

$$
\Delta\left(\theta_{s}, \theta_{v}, \varphi\right)=\sqrt{\tan ^{2} \theta_{s}+\tan ^{2} \theta_{v}-2 \tan \theta_{s} \tan \theta_{v} \cos \varphi} .
$$

[28] This expression quantifies the horizontal distance between the incoming and reflected rays that intersect at level $z$. Although we agree with this expression for the particular case of horizontal leaves, we argue in Appendix A that, in the more general case of spherical distributions, the decorrelation length $d$ must not be measured along the horizontal as in those models, but rather perpendicular to the direction bisecting the Sun and view directions [Hapke, 1986]. The decorrelation length $d$ is then related to the phase angle $\xi$ as

$$
d=\frac{2(z-t)}{\mu_{s}+\mu_{v}} \xi
$$

[29] Adopting equation (7), the transmission corrected for the correlation of the downwelling and upwelling paths becomes

$$
T^{\Downarrow \Uparrow}\left(z, \theta_{s}, \theta_{v}, \xi\right)=\exp \left[\frac{-1}{2}\left(\frac{1}{\mu_{s}}+\frac{1}{\mu_{v}}\right) \int_{0}^{z} u_{L}(t)\left(1-\frac{1}{2} S\left(\frac{z-t}{K}\right)\right) d t\right],
$$

with

$$
K=D \frac{\mu_{s}+\mu_{v}}{2 \xi}
$$

[30] The transmission is then used to derive the reflectance $R_{S}$ that results from a single interaction, either on a foliage element or at the surface [Ross, 1981]:

$R_{S}=\rho_{\text {leaf }} \int_{0}^{H} \frac{u_{L}(z)}{3 \mu_{s} \mu_{v}} T^{\Downarrow \Uparrow}\left(z, \theta_{s}, \theta_{v}, \xi\right) d z+\rho_{\text {surf }} T^{\Downarrow \Uparrow}\left(H, \theta_{s}, \theta_{v}, \xi\right)$, where $\rho_{\text {leaf }}$ is the reflectance from an individual leaf, $\rho_{\text {surf }}$ is the surface reflectance, and $H$ is the canopy depth.

[31] We now study the particular case of a thick homogeneous canopy of leaf area index $L\left(u_{L}(z)=L / H\right.$ with $\left.L \gg 1\right)$. The soil contribution is then negligible. The amplitude of the hot spot $\Delta R_{H S}$ is given by the difference of the modeled reflectance when accounting for the correlated transmission $(S=1)$ and without accounting for this effect $(S=0)$. Integration of equations (8) and (10) shows that

$$
\Delta R_{H S}=\frac{1}{2}\left(R_{S}\right)_{\xi=0}=\frac{\rho_{\text {leaf }}}{3 \mu_{s}} .
$$

[32] The amplitude of the hot spot is half the single scattering contribution at its center. Far from the backscattering direction, the incoming and emerging transmissions are not correlated $(S=0)$, but the single scattering contribution is still significant. Another prediction from this simple modeling is that the hot spot amplitude should vary inversely to the cosine of the solar zenith angle, in agreement with previous modeling results [e.g., Ross, 1981].

[33] Integration of equations (8) and (10) yields

$$
R_{s}=\frac{2 \rho_{\text {leaf }}}{3\left(\mu_{s}+\mu_{v}\right)} \int_{0}^{\infty} \frac{\xi_{m}}{\xi} \exp \left[-\frac{\xi_{m}}{\xi} \int_{0}^{x} 1-\frac{S(r)}{2} d r\right] d x
$$

with $\xi_{m}=\frac{L D}{H} \frac{\left(\mu_{s}+\mu_{v}\right)^{2}}{4 \mu_{s} \mu_{v}} \approx \frac{L D}{H}$. Note that the approximation for $\xi_{m}$ is valid to better than one percent within the hot spot geometry $\left(\xi \leq 10^{\circ}\right)$.

[34] There is no analytical solution for the integrals in equation (12). However, we found that a very accurate approximation can be obtained, in the case of an isotropic distribution of leaves, by

$$
R_{s}=\frac{2 \rho_{\text {leaf }}}{3\left(\mu_{s}+\mu_{v}\right)}\left[1+\frac{1}{1+\alpha \xi / \xi_{m}}\right]
$$

with $\alpha=6.4$ and $\xi$ expressed in radians. Figure 4 shows the integral of equation (12) together with its analytical fit of equation (13).

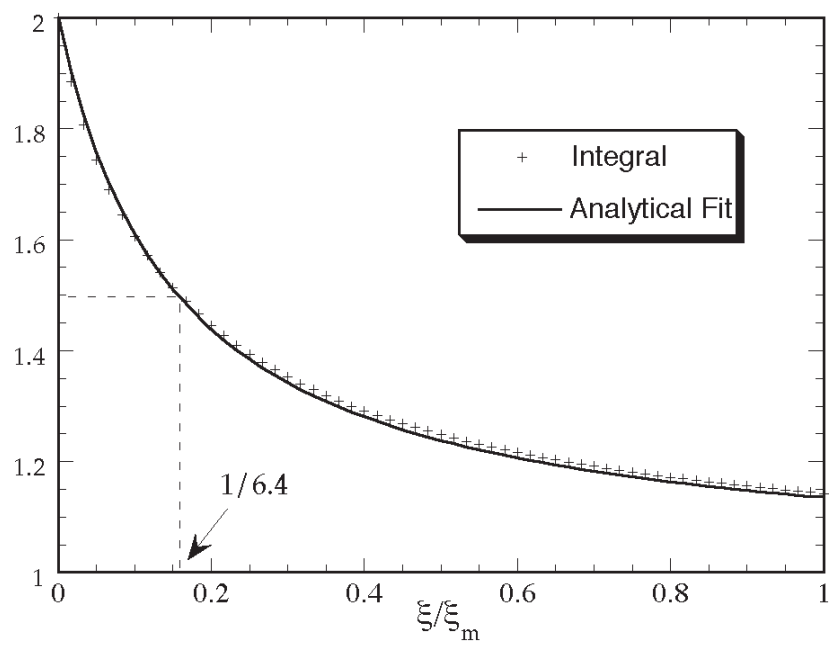

Figure 4. Result of the integration used in equation (12) $I\left(\frac{\xi}{\xi_{m}}\right)=\int_{0}^{\infty} \frac{\xi_{m}}{\xi} \exp \left[-\frac{\xi_{m}}{\xi} \int_{0}^{x} 1-\frac{S(r)}{2} d r\right] d x$ and its analytical fit $F\left(\frac{\xi}{\xi}\right)=1+\frac{1 \xi \xi}{1+\alpha \xi \xi}$. The integration assumes an overlap function $S$ derived for a set of circular leaves randomly oriented. 


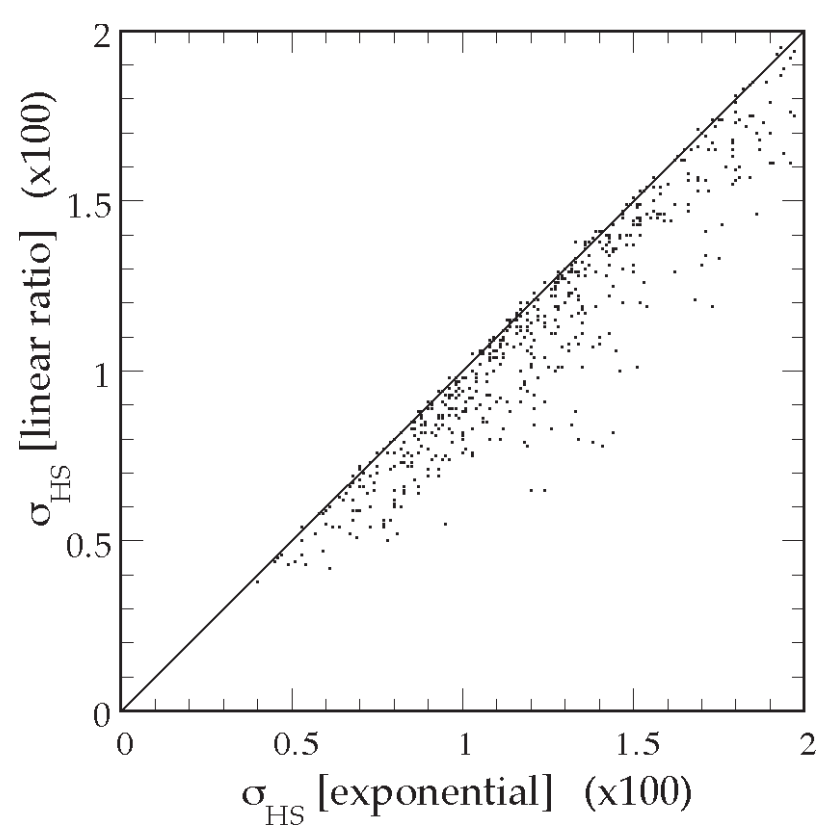

Figure 5. Scatterplot of the RMS error $\left(\sigma_{\mathrm{HS}} \times 100\right)$ around the best fits where the measurements are fitted using either a negative exponential, or a linear ratio as in equation (2). The corresponding measurements were acquired at 865 $\mathrm{nm}$. A selection of the hot spot signatures with an amplitude greater than 0.1 was made.

[35] This theoretical expression for the reflectance generated by single reflection and transmission within the canopy guided the choice for the analytical function to be used for the fit (equation (2)). Within a few degrees around backscattering, the reflectance variations induced by $\mu_{\mathrm{v}}$ are expected to be small compared with those induced by the phase angle. Thus we expect the hot spot reflectance increase close to backscattering to follow:

$$
R_{H S}=\frac{\rho_{\text {leaf }}}{3 \mu_{s}} \frac{1}{1+\xi / \xi_{0}},
$$

where $\xi_{0}$ is the half width at half maximum.

\section{Statistical Analysis}

\subsection{Directional Signature}

[36] In a first step, we verify the validity of using a simple linear ratio to represent the hot spot directional signature. This choice was based on the theoretical development above. On the other hand, several authors suggest a negative exponential $\exp \left(-\xi / \xi_{0}\right)$ to model the sharp reflectance increase toward backscattering. To compare the two approaches, we have fitted the full set of hot spot measurements using either the linear ratio as in equation (2) or a negative exponential. The quality of the fit is quantified by the RMS error $\sigma_{H S}$. It is the result of several error sources, including instrument noise and spatial heterogeneity that are expected to be similar for the two functions. Figure 5 clearly demonstrates that the linear ratio generally yields a significantly better fit than the exponential does. It is a strong argument in favor of the simple theory above. Of course, many other choices are possible and there is no demonstration that the linear ratio is the best, or that it may fit the hot spot signature from all surface types. Nevertheless, the measurements show that a typical shape of the hot spot is as predicted by the simple theory, rather than a mere exponential. Note that, for a given decrease rate at the origin, the exponential predicts a much more rapid reflectance decrease as the phase angle increases than the linear ratio does.

\subsection{Phase Angle Versus Delta}

[37] We now verify the validity of using the phase angle $\xi$ as the dependent variable of the hot spot directional signature. Figure 2 clearly shows that, in many cases, $\xi$ is suitable as the dependent variable. The theory discussed above and the discussion of Appendix A predict that the reflectance should be a function of $\xi$. However, as was also pointed out above, most theoretical models of the hot spot signature indicate that the reflectance should, instead, be a function of $\Delta$ (see equation (6)). Besides, a theoretical canopy composed of planar leaves is expected to generate a hot spot signature that is a function of $\Delta$. Note that $\xi$ and $\Delta$ are strictly equivalent for a Sun at nadir, and that they become uncorrelated as the solar zenith angle increases. Close to the backscattering direction, $\xi$ varies between $\Delta \cos$ $\theta_{s}\left(\right.$ when $\left.\theta_{v}=\theta_{s}\right)$ and $\Delta \cos ^{2} \theta_{s}$ (in the principal plane).

[38] We computed second degree polynomial fits of the measured reflectances for the $7 \times 7$ selected pixels as a function of both $\xi$ and $\Delta$. Three months of measurements were used for this exercise (November, February, and June). At this point, we only make use of the measurements acquired within $4^{\circ}$ of the backscattering direction (from a single POLDER snapshot). The RMSE around the best linear fit, $\sigma$, quantifies the fit quality. $\sigma$ contains several sources of errors, including the spatial variability of the surface and measurement noise, which are expected to be similar for the two proxies. Figure 6 shows a scatterplot of the $\sigma$ values using the two fits. This figure indicates a better

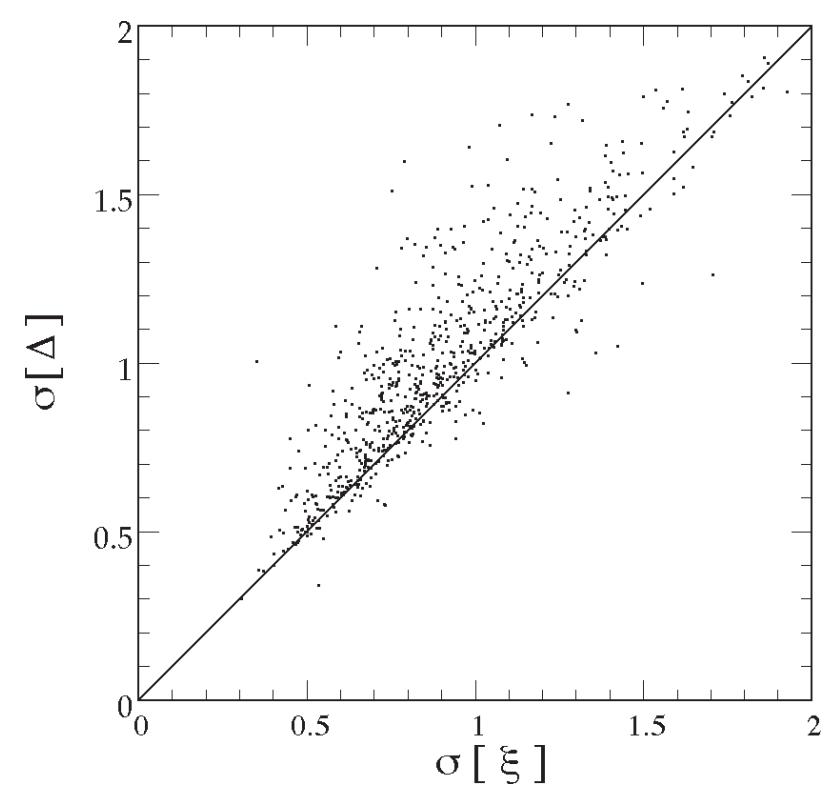

Figure 6. Scatterplot of the RMS error $\left(\sigma_{\mathrm{HS}} \times 100\right)$ around the best fits where the measurements are fitted as a function of phase angle $\xi$ or $\Delta$ (see text). The corresponding measurements were acquired at $865 \mathrm{~nm}$. A selection of the hot spot signatures with an amplitude greater than 0.1 was made. 


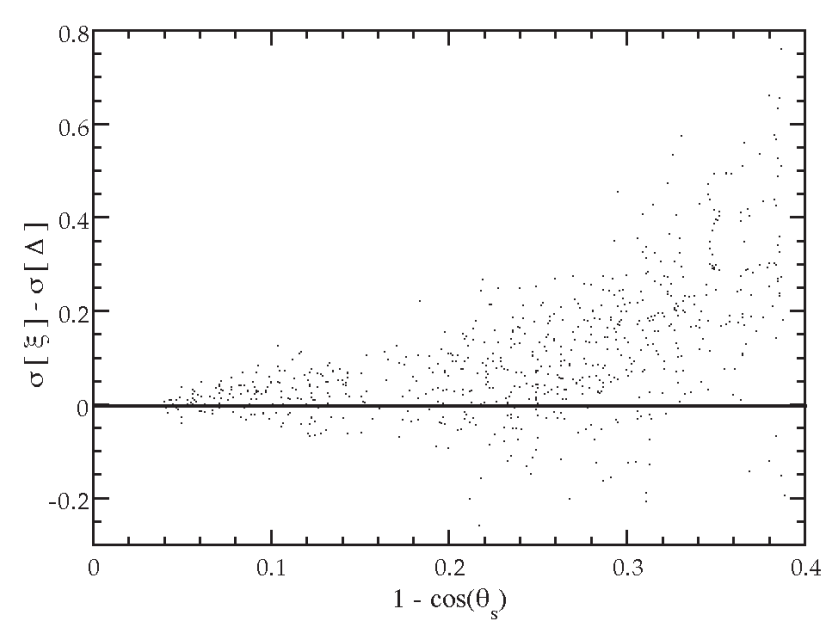

Figure 7. Same measurements as in Figure 6. The difference $\sigma[\Delta]-\sigma[\xi]$ is plotted as a function of $\left(1-\cos \theta_{s}\right)$.

quality for the fits as a function of $\xi$, as expected from the simple theory above. Note that the two choices of variable are expected to yield similar results for small $\theta_{s}$, with increasing differences as a function of $\left(1-\cos \theta_{s}\right)$. Figure 7 shows the difference of the two fit RMSEs as a function of this quantity. The difference increases with $\theta_{s}$. It confirms that the fit quality behaves as expected and that the hot spot signature is generally better defined from the phase angle than from the parameter $\Delta$. Although certainly imperfect, the uniform distribution of leaf orientations appears to be a better approximation of the real world than the planar distribution. We have analyzed plots such as Figures 6 and 7 as a function of surface type, as defined by the IGBP classification. None of the IGBP classes shows a significantly better fit when expressed as a function of $\Delta$ rather than the phase angle $\xi$.

\subsection{Empirical Modeling}

[39] We now present the results obtained with the nonlinear fit described in equation (2). The fit has been applied on the measurements acquired with a signed phase angle between $-50^{\circ}$ and $4^{\circ}$ (see Figure 2). According to the theoretical section above, we expect that the half width $\xi_{0}$, expressed in radians, and the amplitude $\mathrm{A}$, be related to the canopy structural and optical parameters by (in the case of deep canopies):

$$
\xi_{0} \approx \frac{D L}{6.4 H} \text { and } \quad \mu_{s} A=\frac{\rho_{\text {leaf }}}{3} .
$$

[40] We have analyzed the range of variation of $\xi_{0}, A$ and $\rho_{\text {leaf }}$. The hot spot signature quality was quantified by the signal-to-noise ratio $\mathrm{A} / \sigma_{\mathrm{HS}}$. This ratio is computed both for 670 and $865 \mathrm{~nm}$, and the best quality targets were selected according to the smallest of the two band values (the signalto-noise ratio is smaller at 670 than at $865 \mathrm{~nm}$ in most cases). For each of the IGBP classes (except, again, Wetlands, Ocean, Snow \& Ice and Urban for lack of suitable targets) the fifteen best cases have been selected and are now discussed. The cases presented in Figure 2 have been extracted from this particular data set. Figure 8 shows the locations of these high quality observations. The selected targets do not cover the Earth evenly. This is to be expected considering that only a few hundred data points are displayed. Also, the selection of homogeneous areas in our processing imposes a strong constraint on their geographical coverage.

\subsection{Spectral Signature}

[41] Equation (15) indicates that the angular half width $\xi_{0}$ is independent of the wavelength. Note that, in principle, this equation is valid only for the deep canopy cases. This independence may be verified from a statistical analysis of our results. Figure 9 shows a scatterplot of the half width at $670 \mathrm{~nm}$ and $865 \mathrm{~nm}$. This figure indicates a few abnormal results, where the width is very different in the two bands. They correspond to forested targets according to the IGBP classification. We have identified a few cases where the width spectral disparity appears significant. Apart from those few points, the results are well distributed along the 1:1 line. The half width of most of the targets is between $1^{\circ}$

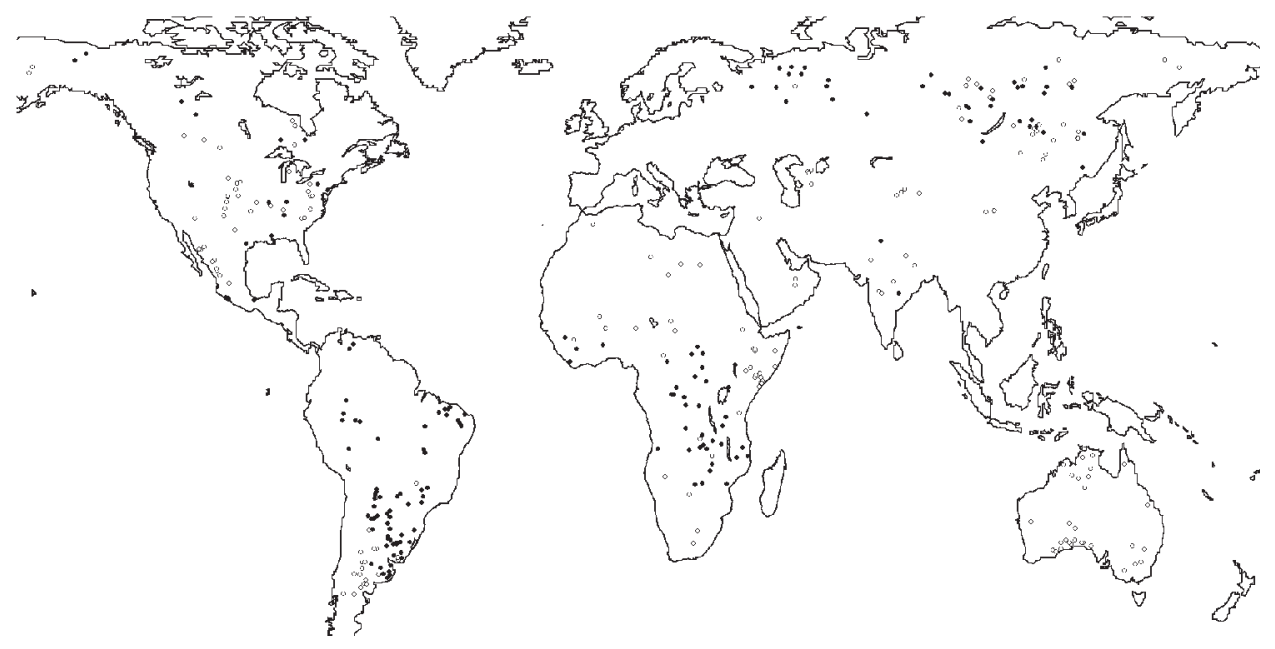

Figure 8. Position of the targets used in the statistical analysis of the hot spot signatures observed from space. These correspond to the 15 targets that yield the best signal-to-noise ratio for each IGBP class. Open circles are the targets used only for width and amplitude estimate. The solid circles correspond to the targets where a leaf reflectance estimate was also made (NDVI >0.6). 


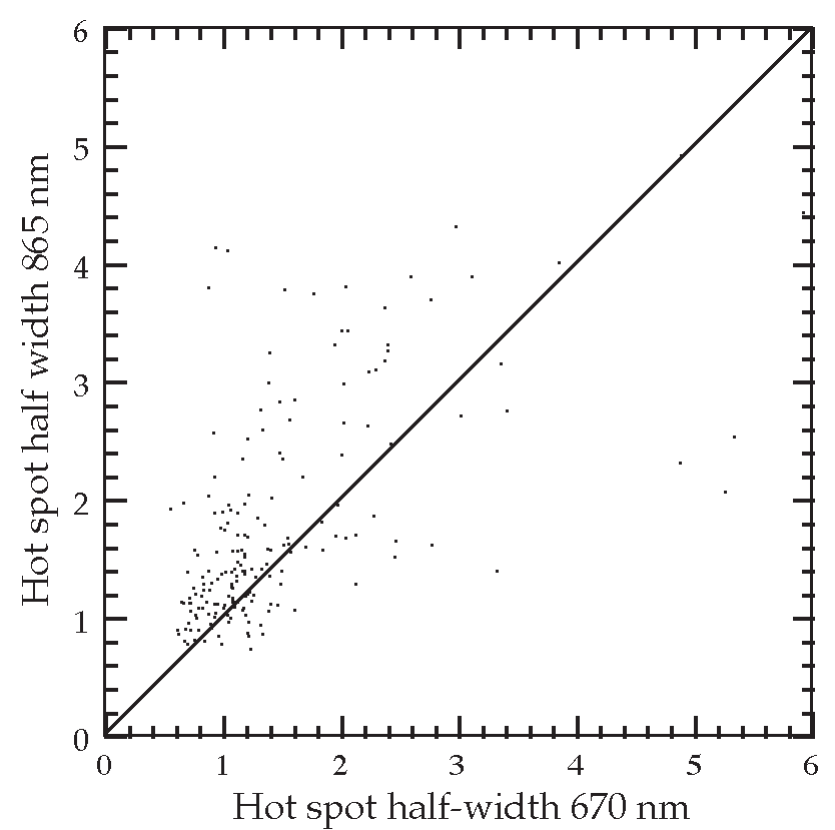

Figure 9. Scatterplot of the hot spot half width $\xi_{0}$ for the $865 \mathrm{~nm}$ band as a function of the same at $670 \mathrm{~nm}$. The data points are the same as those used for Figures 10 and 11.

and $2^{\circ}$, with a range of variation of $1^{\circ}-6^{\circ}$. Considering only those data points with a half width lower than 2 in the two bands, the scatter is significant in regard to the unexpectedly small variability. The estimated width uncertainty of a few tenths of a degree is consistent with the observed scatter (the standard deviation of the width difference is 0.34 ). There is a slight bias of the $865 \mathrm{~nm}$ width compared to that at 670 $\mathrm{nm}$ that amounts to $0.2^{\circ}$. We have no explanation for this small bias. Because the signal-to-noise ratio is generally larger at $865 \mathrm{~nm}$ (the hot spot signal is larger, with less atmospheric perturbation), we have more confidence in the near infrared estimates of the width.

\subsection{Hot Spot Width as a Function of Surface Type}

[42] Figure 10 shows the measured widths as a function of the IGBP surface classification for 670 and $865 \mathrm{~nm}$. The

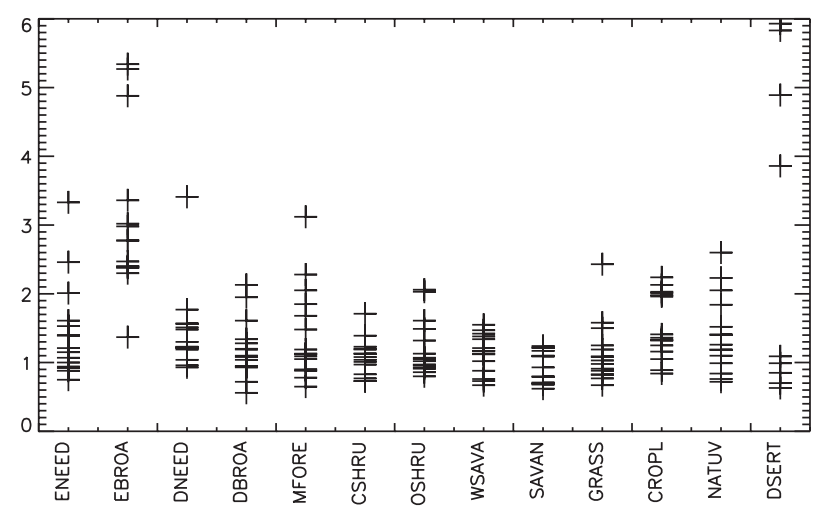

classes that show some dispersion of half width, in the range $0.8^{\circ}-4^{\circ}$, are the forest classes (Broadleaf and Needleleaf, both Evergreen and Deciduous, and Mixed Forest) and the Desert class. The "evergreen broadleaf" class elements tend to have larger half widths than other classes. This result is consistent with the large leaf sizes of the trees in tropical forests (see equation (15)). Other forest classes show some variability, but a large fraction of the targets show half widths of $0.8^{\circ}-2^{\circ}$. Elements of all other classes (i.e., nonforest and nondesert) have half widths in the range $0.8^{\circ}-2^{\circ}$ with no significant separation between the classes. Except for the evergreen broadleaf case, the variability within the classes appears larger than the variation of the mean (or median) between the classes. It appears that the hot spot width is not an effective indicator of the vegetation IGBP classification, and that it is sensitive to canopy parameters that are more variable within the IGBP classes than between these classes. This makes the interpretation of the retrieved width in terms of vegetation structural parameters rather difficult.

[43] Our procedure does not attempt to capture all the variability of the various biomes. The selection of spatially homogeneous surfaces may reject a variety of vegetation types. Moreover, the search for high signal-to-noise ratio tends to select targets with a large amplitude hot spot signature. We cannot identify why our method would favor a given range of width, but this hypothesis cannot be ruled out.

\subsection{Hot Spot Amplitude and Leaf Reflectance}

[44] Figure 11 shows the hot spot amplitude as a function of the surface type. Desert surfaces show a rather large dispersion of hot spot amplitude. This observation confirms the visual impression from plots such as those of Figures $2 n-2 p$. The Evergreen Broadleaf case clearly stands out from the other vegetated surfaces with a very small amplitude at $670 \mathrm{~nm}$ and a rather large dispersion at $865 \mathrm{~nm}$, as illustrated by Figures $2 \mathrm{~b}$ and $2 \mathrm{c}$. Other classes do not show specific features in the amplitude distribution. At $865 \mathrm{~nm}$, the amplitude varies between 0.10 and 0.20 in most cases. Note that our selection of high signal to noise may reject the cases with small amplitudes. There is more relative variation at $670 \mathrm{~nm}$ with some separation between the classes. In general, the amplitude appears smaller for the forest classes

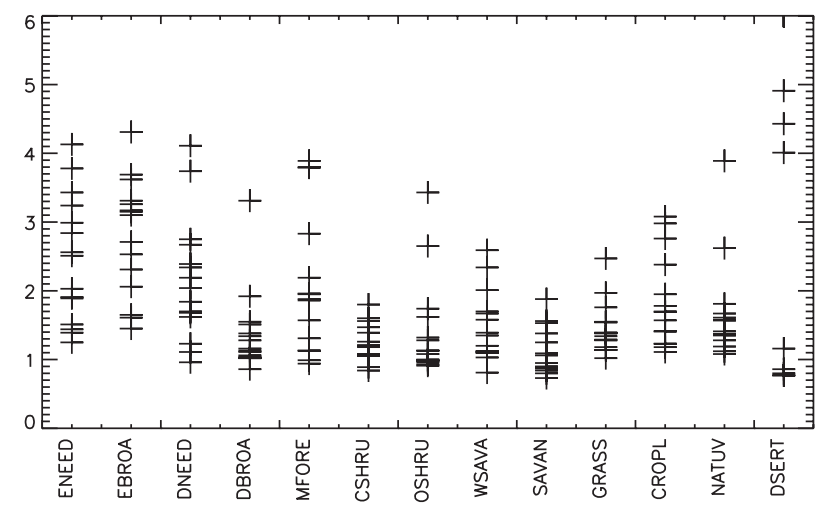

Figure 10. Hot spot half width $\xi_{0}$ as a function of the IGBP surface classification at (left) $670 \mathrm{~nm}$ and (right) $865 \mathrm{~nm}$. The 13 classes that are used here are "evergreen needleleaf forest", "evergreen broadleaf forest", "deciduous needleleaf forest", "deciduous broadleaf forest", "mixed forest", "closed shrubland", "open shrubland", "woody savanna", "savanna", "grassland", "cropland", "cropland/ natural vegetation mosaic", and "barren or sparsely vegetated". 

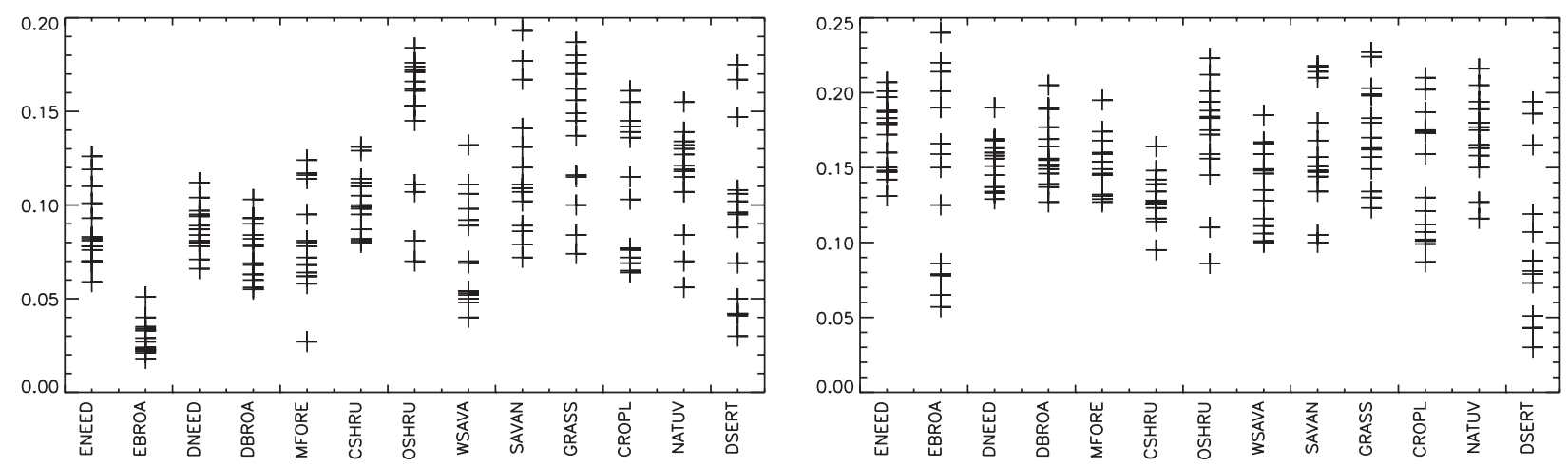

Figure 11. Same as Figure 10 but for the hot spot Amplitude $A$.

than for the others. This may be interpreted as a background contribution to the reflectance for nonforest cases.

[45] Equation (15) indicates that, assuming a deep canopy (negligible surface contribution), the amplitude is proportional to the leaf reflectance with a dependence on the solar zenith angle. Therefore, for a given target, some variation of the amplitude is expected with the solar zenith angle. In Figure 12, we have selected a new set of suitable targets limited to those having a large spectral signature, indicative of photosynthetic activity. Selection is made on the widely used Normalized Difference Vegetation Index (NDVI), computed from the retrieved B (see equation (2)), with a threshold value of 0.6 . There is no suitable target for the desert class and only a few for other arid type classes. The leaf reflectance is computed as in equation (15). The order of magnitude of the leaf reflectance at 865 $\mathrm{nm}$ is on the order of 0.4 , which is somewhat smaller than the expected values $(0.5)$ from laboratory measurements [e.g., Jacquemoud and Baret, 1990]. This low value may be the result of the contribution of foliage elements other than leaves to the reflectance or of the soil and litter background. Another potential explanation is the anisotropy of leaf reflectance when the simple models assume a Lambertian leaf. The directional signature of the leaf reflectance may change the ratio between $\rho_{\text {leaf }}$ and $A$. The retrieved leaf reflectance shows more variability at $670 \mathrm{~nm}$ with significant differences between some of the IGBP classes. The Evergreen Broadleaf class consistently shows very small reflectances, on the order of 0.05 . For other classes, the retrieved leaf reflectance is generally between 0.1 and 0.2 .

[46] Note that the scatterplots of leaf reflectance show less variability within classes than the amplitude ones. This is due in part to the selection for high vegetation cover. Nevertheless, when comparison is made using the same set of data, we do observe a lower relative variability in leaf reflectance than in hot spot amplitude. This result indicates that, as expected, the hot spot amplitude increases with the solar zenith angle.

\section{Discussion}

\subsection{Atmospheric Effects}

[47] The scattering phase function of aerosol sometimes shows a significant maximum for a scattering angle of $180^{\circ}$, in particular for large particles such as dust. This local maximum in the phase function may be narrow. Thus aerosol scattering may produce a reflectance maximum in the backscattering direction, which would be mistakenly interpreted as a surface signature. Such an atmospheric signature is expected to be roughly proportional to the optical thickness and therefore to somewhat increase toward shorter wavelength. On the other hand, the surface contribution is proportional to the leaf reflectance, which is small in the visible, both at 440 and $670 \mathrm{~nm}$. Therefore, if a hot spot peak due to aerosols was present, it should lead to a
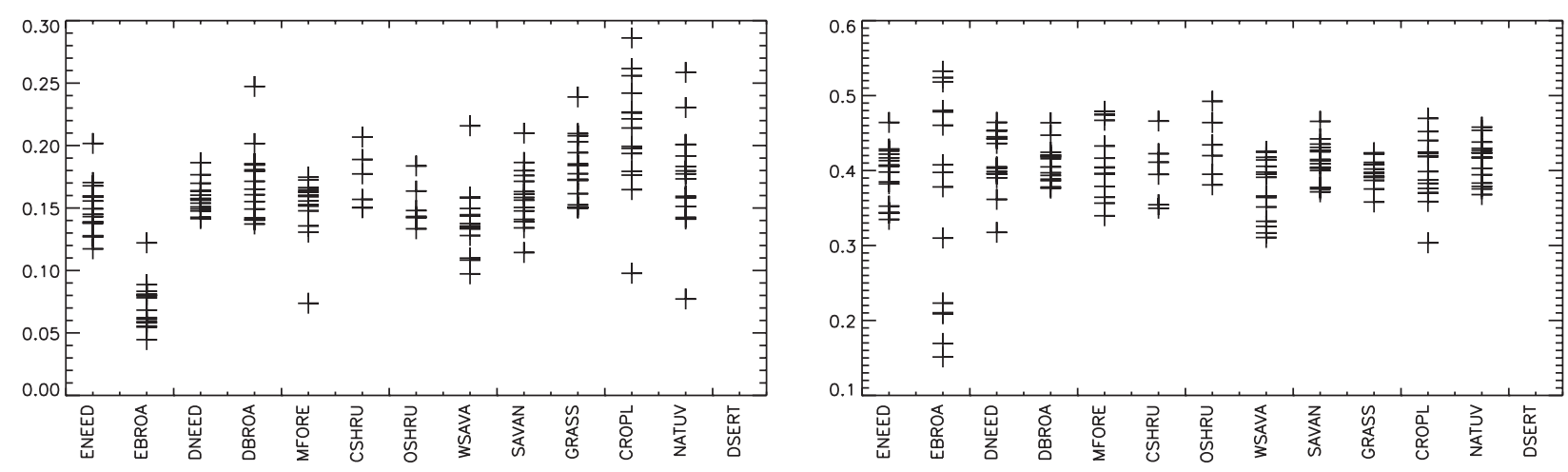

Figure 12. Same as Figure 10 but for the leaf reflectance. The leaf reflectance was derived from the hot spot amplitude using equation (15). A more restricted set of targets than for Figures 10 and 11 was used to ensure a large vegetation cover (NDVI $>0.6)$. 
larger reflectance peak at $440 \mathrm{~nm}$ than at $670 \mathrm{~nm}$. We have examined a very large number of hot spot signatures at 440 , 670 , and $865 \mathrm{~nm}$. We have not been able to identify a single case where the hot spot magnitude appears larger at the shorter wavelength. This result indicates that aerosols with a significant maximum at backscattering are not prevalent.

[48] To further ascertain this claim, we have analyzed a few POLDER images acquired over the ocean during large dust events. Dust is composed of rather large particles and is therefore the most likely aerosol to generate a backscatter feature that would contaminate our analysis. We looked for a local maximum of the reflectance in the backscattering direction. None of the cases that we have analyzed show such signature. This provides further evidence that the reflectance maximum at backscattering is solely generated at the surface.

[49] The reflectance directional signatures have been corrected for the atmosphere accounting only for the molecular scattering to first order. No correction was made for the effect of aerosol scattering or the coupling of surface reflectance and molecular scattering. Therefore the absolute values of the reflectances may not be fully representative of the surface. The atmospheric scattering generates rather smooth directional signatures. Thus the contribution to the TOA reflectance that went through atmospheric scattering does not affect the retrieved $A$ and $\xi_{0}$ in our statistical analysis. On the other hand, the reduced atmospheric transmission due to the aerosol load may lead to an underestimate of $A$ and, in turn, the leaf reflectance. No significant effect is expected on the retrieved width, as was pointed out by Powers and Gerstl [1988]. For an aerosol optical thickness of 0.1 , the underestimate of $A$ and/or the leaf reflectance would be on the order of $30 \%$, depending on the solar zenith angle. This may be a factor for the variability of the retrieved $A$ and $\rho_{\text {Leaf }}$.

\subsection{Spatial Scale}

[50] POLDER data have been acquired at a resolution of $6 \mathrm{~km}$. Our analysis assumes that each pixel is statistically similar to its neighbors over an area of roughly $50 \times 50$ $\mathrm{km}^{2}$. Note that it is not necessary that a given pixel be homogeneous at all scales below the pixel scale. There may be some heterogeneity within a pixel provided that the relative fractions of each component are fairly constant over the 49 POLDER pixels. For example, the "wooded savanna" landscape includes grass and trees, the fraction of which may be constant at the POLDER pixel scale, over tens of kilometers. Most certainly, the homogeneity constraint is rather strong in our processing. On the other hand, the use of measurements far from the hot spot direction gives us the ability to quantify this homogeneity and to select suitable pixels. For the analysis presented in this paper, we were able to examine a large number of cases with the hot spot geometry, and to reject most of them based on objective criteria.

[51] It is clear that a surface homogeneous at all scales between a given scale and $6 \mathrm{~km}$ will provide exactly the same hot spot feature for any scale of observation between these two scales. On the other hand, the relatively coarse spatial resolution of the POLDER pixels probably smoothes the natural variability of hot spot features, as most surfaces are heterogeneous at all scales of observation. The hot spot feature variability is expected to increase as the size of the pixels decreases.

\subsection{Hot Spot and Albedo}

[52] A question of importance is whether the hot spot directional signature must be accounted for when estimating the surface albedo. Assuming that the hot spot amplitude is equal to the mean surface reflectance, and that the half width is $2^{\circ}$, a rough estimate shows that the hot spot contains less than one thousandth of the reflected flux. This, together with the results of half widths outlined in this paper, strongly suggests that most applications do not need to account for the narrow hot spot signature in an evaluation of the surface albedo.

\subsection{Directional Resolution}

[53] Theoretical considerations indicate that the angular width of the hot spot is related to $L D / H$. Putting typical numbers into equation (15) indicates that the angular width of hot spot signatures generated by small leaves distributed over tall canopies is smaller than the angular resolution of POLDER. Therefore one may argue that this instrument is not well suited for the measurements of the hot spot signature and that another design would measure signatures with a better angular resolution. However, let us point out that the angular width of the Sun is slightly larger than $0.5^{\circ}$. Therefore any theoretical angular signature with a scale smaller than this value will be smoothed out because of the angular spread of the input radiance. The angular resolution of POLDER is slightly better than the sun width. This resolution appears optimal for the purpose of angular signature measurements over natural targets.

\subsection{Terminology}

[54] The analysis of the directional signature of surface reflectance has received much interest in recent years. All measurements and models show an increase of reflectance toward the backscattering direction. This increase is often referred to as the hot spot effect. However, due to the limited angular resolution and/or sampling of the measurements, the large increase of the reflectance in the very few degrees around backscattering is not observed. There is no "spot" in the directional signature that is measured, but rather a very broad and smooth reflectance increase. Such a signature may be explained by the large-scale structure of the canopy, i.e., the shadowing by opaque objects such as tree crowns or rocks. It may be modeled by geometric optics and does not involve porous media. On the other hand, the process that involves the penetration of light within the canopy or soil yields a much narrower reflectance peak, which may be designated as a spot. We believe that the two processes should not be referred to by the same terminology, and that "hot spot" should be reserved for the reflectance peak observed within a few degrees of the backscattering direction, which is generated by radiative transfer within the foliage or soil.

\subsection{Coherent Backscatter}

[55] It is widely expected that shadow hiding is the dominant cause of the hot spot in vegetation. Thus hot spot modeling has concentrated on leaves and needles and larger structures. However, processes at all scales from a wave- 
length to a pixel can potentially influence an angular reflectance signature. Coherent backscatter is a wavelength-scale hot spot mechanism that is important for lunar soil and other barren solar system surfaces [Hapke et al., 1993]. The very few measurements of the relative importance of shadow hiding and coherent backscatter for terrestrial vegetated surfaces suggest that while shadow hiding dominates for most plants and clumpy moist soils, coherent backscatter can dominate for finely structured plants such as moss and for fine dry soils [Hapke et al., 1996]. Laboratory studies indicate that coherent backscatter generates a peak of width less than $0.5^{\circ}$ varying proportionally to wavelength [Hapke et al., 1993]. The present paper reports half widths larger than $1^{\circ}$ and roughly spectrally neutral, which do support the idea that shadow hiding mechanism is prevalent on terrestrial surfaces.

\subsection{TRIANA}

[56] One objective of the TRIANA spacecraft that will observe the Earth from the vicinity of the Lagrange L1 point is a detailed analysis of the reflectance directional signature close to backscattering. L1 is located between Earth and the Sun and the phase angle of observation is close to zero for any point on the Earth. On the other hand, direct communication with the spacecraft is not possible when it is located on the L1 point because of solar noise. Therefore TRIANA will orbit L1 and the phase angle of observation will vary between $4^{\circ}$ and $15^{\circ}$. Our results indicate that most surfaces display a hot spot limited to a range of phase angle smaller than $2^{\circ}$. The directional signature that will be sampled by the TRIANA mission is not the hot spot as defined in the present paper, but rather the broad reflectance increase toward backscattering that is observed from in situ, airborne or spaceborne measurements. A spaceborne instrument designed to monitor the backscatter directional signature should, for a complete picture, measure the strong and narrow hot spot we have described by sampling the reflectance for phase angles between $0^{\circ}$ and $4^{\circ}$ with an angular sampling better than $0.5^{\circ}$.

\section{Summary and Conclusion}

[57] The POLDER instrument allows the measurement of the hot spot directional signature with an unprecedented angular resolution provided that a fairly homogeneous area can be found in the data. We have processed eight months of spaceborne measurements and extracted the directional signature of the pixels that were observed in the backscattering direction. The large number of such signatures allows an evaluation of the predictions made by simple modeling of the radiative transfer within the canopy.

[58] The reflectance close to the backscattering direction is a function of the phase angle. This parameter allows a better fit through the measurements than a parameter $\Delta$, used by several models of the hot spot reflectance, that quantifies the horizontal distance between the Sun and view directions. This is because some of these models are based on an assumption of horizontally oriented leaves [e.g., Jupp and Strahler, 1991], when a spherical distribution appears to be a better approximation. Other models that do not specifically assume horizontal leaves incorrectly estimate the intersection of canopy gaps on the incoming and outgoing paths over the horizontal rather than perpendicular to the sun rays [e.g., Nilson and Kuusk, 1989; Verstraete et al., 1990; Qin, 1993].

[59] Simple modeling of the radiative transfer within the canopy indicates that the hot spot directional signature is proportional to $\left(1+\xi / \xi_{0}\right)^{-1}$. The measurements confirm this prediction. In fact, the observed signatures are better fitted with this function than with a negative exponential that is often used for hot spot modeling.

[60] The same simple modeling indicates that the hot spot width is independent of wavelength and proportional to $L D / H$ where $D$ is a typical scale of the foliage elements, $H$ the thickness of the foliage layer and $L$ is the LAI (only for large LAI). Most of the measurements show an unexpectedly small range of variation of the hot spot half-width, between $0.8^{\circ}$ and $2^{\circ}$. Some dispersion occurs for the cases belonging to the forest and desert IGBP classes, in the range $1^{\circ}-4^{\circ}$. The width appears to be spectrally neutral in accordance with theory, although the noise from our procedure is significant in regard to the small range of variation.

[61] No significant hot spot signature has been observed when the surface reflectance is very small, as in the blue channel or over the ocean. This result indicates that the atmospheric contribution to the reflectance increase at the backscattering direction is negligible.

[62] An analysis of the retrieved width as a function of the IGBP classification shows a variability within the classes that is significantly larger than the difference between the class centers. Besides, the variability of the hot spot width is much smaller than expected from modeling [Qin and Goel, 1995; Qin et al., 1996; Chen and Leblanc, 1997]. The hot spot amplitude is generally on the order of $0.10-0.20$ at $865 \mathrm{~nm}$, and $0.05-0.20$ at 670 $\mathrm{nm}$, although the full range of values is wider (the amplitude is very small at $670 \mathrm{~nm}$ over evergreen broadleaf forests). For thick canopies, it may be interpreted in terms of foliage element (leaf) reflectance. Retrieved values are on the order of 0.4 in the near infrared and show more variability at $670 \mathrm{~nm}$ : on the order of 0.05 for "evergreen broadleaf" targets, and roughly between 0.14 and 0.20 for other surface types.

\section{Appendix A}

[63] To our knowledge, the only directional reflectance models that describe the hot spot signature as a function of the angular variable $\xi$ are the works of Hapke [1986] and Chen and Leblanc [1997]. Most models make use of $\Delta$ as the angular variable rather than the phase angle that is used in the present paper. Apparently, this may be traced back either to the work by Jupp and Strahler [1991] or to Verstraete et al. [1990] or to Kuusk [1985].

[64] In the work of Jupp and Strahler [1991], a very general theory is developed. It demonstrates that the hot signature is related to the relative overlap of foliage elements projected over an horizontal plane along the Sun and view directions (their equation (4)). Then, the particular case of planar leaves is analyzed. It is shown that, for this particular case, the overlap is a function of $\Delta$. Nothing is said about other cases, such as a spherical distribution of leaves. Nevertheless, it seems that many authors have extended the planar leaves result to the general case with 


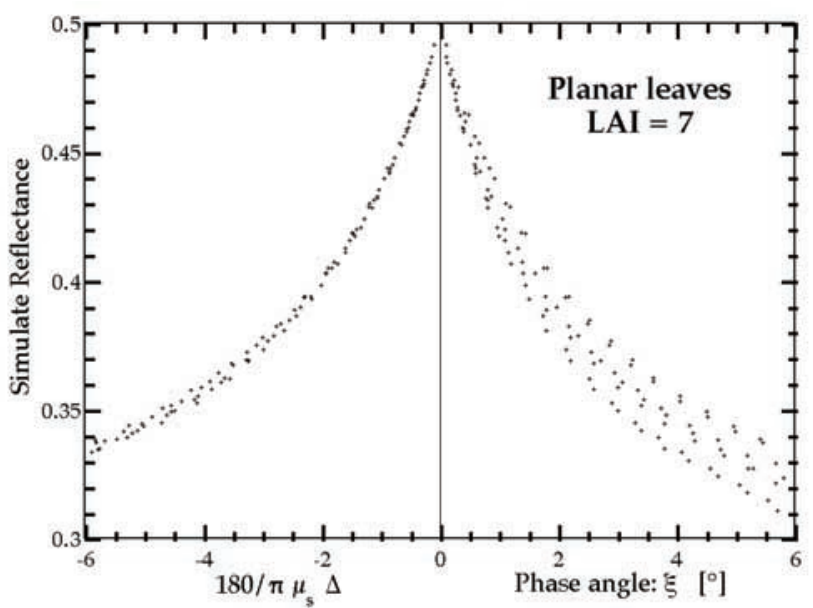

Figure A1. Result of Monte Carlo simulation of the reflectance generated by a single interaction of photons with a canopy or the surface. The results are shown both as a function of the phase angle (right side of the figure) and $\Delta$ (left side of the figure). In order to have comparable scales, $\Delta$ has been multiplied by a constant value of $180 \mu_{\mathrm{s}} / \pi$. In this figure, the leaf orientation is planar, and the LAI is 7.

no justification. When one considers leaves that are not horizontal, the projected surfaces on a horizontal plane (along the Sun and view directions) differ. Their overlap is then not a simple function of $\Delta$. On the other hand, numerical experiments clearly demonstrate that, for a spherical distribution of leaves, on average the projected surface overlap scales with the phase angle rather than $\Delta$.

[65] Verstraete et al. [1990, p. 11,758] argues that a distribution of leaves with arbitrary orientation may be replaced by equivalent planar leaves. The theory is then developed for such planar leaves, which yield a hot spot function that is function of $\Delta$. However, in the context of joint probability of penetrating and reflected paths, the two orientations are not equivalent, as is demonstrated below. Thus the modeling error can be traced back to this assumption.

[66] Kuusk [1985] analyzes the joint probability of penetrating and reflected paths. The probability of going

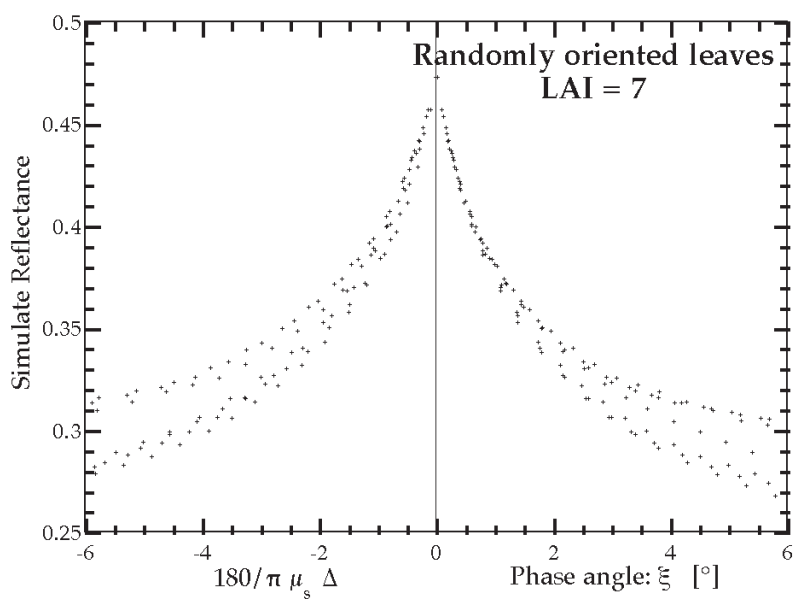

Figure A2. Same as Figure A1 but with a random orientation of leaves.

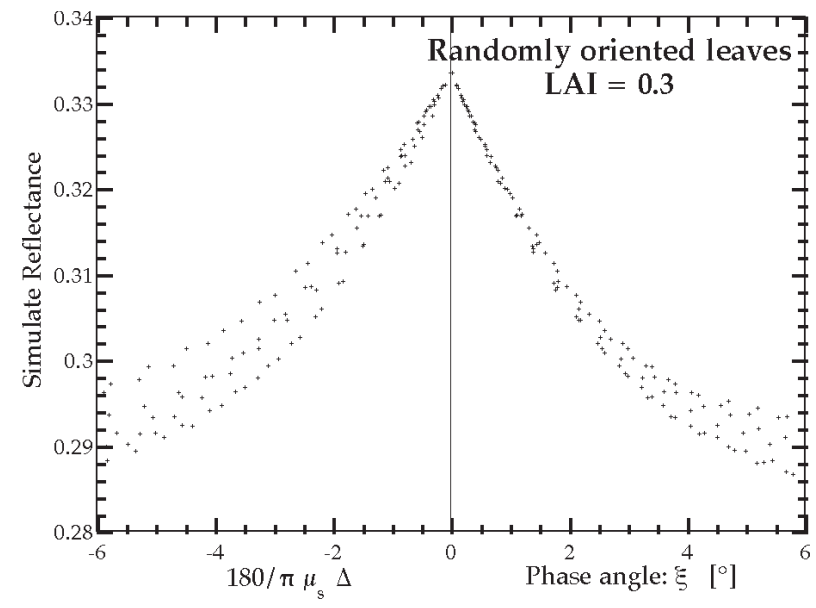

Figure A3. Same as Figure A1 but with a random orientation of leaves and a LAI of 0.3 .

through a given layer of leaves is determined from the distance between the penetrating and reflected directions at the considered level. This distance is computed over the horizontal. Let us point out that the probability of interest concerns individual leaves (the position of leaves is assumed randomly distributed, and their respective positions are therefore uncorrelated). For leaves of random orientation, the horizontal is not a relevant direction. We argue that the distance should rather be computed perpendicular to the Sun and view directions (these directions are very close in the hot spot). Again, for the particular case of planar leaves, we agree that the correlation distance shall be computed in the horizontal plane that is a relevant direction.

[67] To further ascertain our claim that theory supports the phase angle rather than $\Delta$ to describe the hot spot, we made Monte Carlo simulations of the radiative transfer within the canopy. Our simulations are based on the exact same hypothesis as the papers referred to above: The canopy is made of an ensemble of leaves randomly distributed within a layer. We only consider photons that are reflected only once either at the surface or by a leaf. In the simulations, the leaves are opaque and their orientation distribution is either planar or spherical. The leaf reflectance is set to 0.5 , and the surface reflectance is 0.2 (near infrared typical values). We have set the dimensionless ratio $\mathrm{D} / \mathrm{H}$ so that the hot spot half width is roughly 1.5 degree, a typical value as shown by POLDER measurements. The solar zenith angle is $45^{\circ}$. The reflectance is estimated over 120 different directions surrounding backscattering. Figures A1 to A3 present the simulation results as a function of both the phase angle $\xi$ and $\Delta$. In order to have comparative scales, $\Delta$ has been multiplied by a constant value of $180 \mu_{\mathrm{s}} / \pi$. Figure A1 is a case with a deep canopy (LAI = 7) and a planar leaf distribution. The figure clearly demonstrates that the data is much better ordered with $\Delta$ as the independent variable and that the hot spot signature is not well represented by $\xi$. Figure A2 is the same deep canopy, but with a spherical leaf distribution. The dispersion is now much larger as a function of $\Delta$ than when using $\xi$ as the variable. This simulation confirms our theory that the hot spot directional signature generated by randomly oriented leaves is a function of the phase angle. The dispersions that are 
observed for larger phase angles result from the variation with solar and zenith angle (see equation (13)). There is no such dispersion on plots that show a corrected reflectance $R_{\text {sim }}\left(\cos \theta_{s}+\cos \theta_{v}\right)$ as a function of $\xi$.

[68] Finally, Figure A3 is the result of a simulation with a thin canopy $(\mathrm{LAI}=0.3)$ of randomly oriented leaves above a Lambertian surface. Again the hot spot signature is better represented by the phase angle than by $\Delta$. As with the deep canopy case, there is some dispersion as the phase angle increases. The simulated reflectance includes surface and canopy contributions that vary differently as a function of the Sun and view angle. In this case, there is no simple correction that reduces the dispersion.

\section{References}

Bicheron, P., and M. Leroy, Bidirectional reflectance distribution function signatures of major biomes observed from space, J. Geophys. Res., 105, 26,669-26,681, 2000.

Bréon, F. M., and S. Colzy, Cloud detection from the spaceborne POLDER instrument and validation against surface synoptic observations, J. Appl. Meteorol., 38, 777-785, 1999.

Bréon, F. M., V. Vanderbilt, M. Leroy, P. Bicheron, C. L. Walthall, and J. E. Kalshoven, Evidence of hot spot directional signature from airborne POLDER measurements, IEEE Trans. Geosci. Remote Sens., 35, 479484, 1997.

Chen, J. M., and S. G. Leblanc, A four-scale bidirectional reflectance model based on canopy architecture, IEEE Trans. Geosci. Remote Sens., 35, 1316-1337, 1997.

Deschamps, P. Y., F. M. Breon, M. Leroy, A. Podaire, A. Bricaud, J. C. Buriez, and G. Seze, The POLDER mission: Instrument characteristics and scientific objectives, IEEE Trans. Geosci. Remote Sens., 32, 598615, 1994.

Gerstl, S. A. W., and C. Simmer, Radiation physics and modelling for offnadir satellite sensing of non-Lambertian surfaces, Remote Sens. Environ., 20, 1-29, 1986.

Goel, N. S., W. Qin, and B. Wang, On the estimation of leaf size and crown geometry for tree canopies from hot spot observations, J. Geophys. Res., 102, 29,543-29,554, 1997.

Grant I. F., et al., First observation of the hot spot from space at sub-degree angular resolution using POLDER data, Int. J. Remote Sens., in press, 2002.

Hapke, B., Bidirectional reflectance spectroscopy, 4, The extinction coefficient and the opposition effect, Icarus, 67, 264-280, 1986.

Hapke, B., R. Nelson, and W. Smythe, The opposition effect of the moon: The contribution of coherent backscatter, Science, 260, 509-511, 1993.

Hapke, B., D. DiMucci, R. Nelson, and W. Smythe, The cause of the hot spot in vegetation canopies and soils: Shadow hiding versus coherent backscatter, Remote Sens. Environ., 58, 63-68, 1996.

Hautecoeur, O., and M. Leroy, Surface bidirectional reflectance distribution function observed at global scale by POLDER/ADEOS, Geophys. Res. Lett., 25, 4197-4200, 1998.

Jacquemoud, S., and F. Baret, PROSPECT: A model of leaf optical properties spectra, Remote Sens. Environ., 34, 75-91, 1990.

Jupp, D. L., and A. H. Strahler, A hot spot model for leaf canopies, Remote Sens. Environ., 38, 193-210, 1991.

Kuga, Y., and A. Ishimaru, Retroreflection from a dense distribution of spherical particles, J. Opt. Soc. Am., A1, 831-835, 1984.

Kuusk, A., The hot spot effect of a uniform vegetative cover, Sov. J. Remote Sens., 3, 645-658, 1985.

Kuusk, A., A fast, invertible canopy reflectance model, Remote Sens. Environ., 51, 342-350, 1995.

Loveland, T. R., and A. S. Belward, The IGBP-DIS global $1 \mathrm{~km}$ land cover data set, DISCover: First results, Int. J. Remote Sens., 18, 3291-3295, 1997.

Myneni, R., and E. T. Kanemasu, The hot spot of vegetation canopies, J. Quant. Spectrosc. Radiat. Transfer, 40, 165-168, 1988.

Nilson, T., and A. Kuusk, A reflectance model for the homogeneous plant canopy and its inversion, Remote Sens. Environ., 27, 157-167, 1989.

Powers, B. J., and S. A. W. Gerstl, Modelling of atmospheric effects on the angular distribution of a backscattering peak, IEEE Trans. Geosci. Remote Sens., 26, 649-659, 1988.

Qin, W., Modeling Bidirectional reflectance of multicomponent vegetation canopies, Remote Sens. Environ., 46, 235-245, 1993.

Qin, W., and N. S. Goel, An evaluation of hotspot models for vegetation canopies, Remote Sens. Rev., 13, 121-159, 1995.

Qin, W., N. S. Goel, and B. Wang, The hotspot effect in heterogeneous vegetation canopies and performance of various hotspot models, Remote Sens. Rev., 14, 283-332, 1996.

Ross, J. K., The Radiation Regime and Architecture of Plant Stands, Dr. W. Junk, Norwell, Mass., 1981.

Roujean, J. L., M. Leroy, and P. Y. Deschamps, A bidirectional reflectance model of the Earth's surface for the correction of remote sensing data, J. Geophys. Res., 97, 20,455-20,468, 1992.

Seeliger, H., Theorie der Beleuchtung staubförmiger kosmischer Massen insbesondere des Saturnringes, Abh. Bayer. Akad. Wiss. Math. Naturwiss., 18, 1-72, 1895.

Vermote, E. F., D. Tanré, J. L. Deuzé, M. Herman, and J. J. Morcrette, Second simulation of the satellite signal in the solar spectrum, 6S: An overview, IEEE Trans. Geosci. Remote Sens., 35, 675-686, 1997.

Verstraete, M. M., B. Pinty, and R. E. Dickinson, A physical model of the bidirectional reflectance of vegetation canopies, 1, Theory, J. Geophys. Res., 95, 11,755-11,765, 1990.

Woessner, P., and B. Hapke, Polarization of light scattered by clover, Remote Sens. Environ., 21, 243-261, 1987.

F.-M. Bréon and F. Maignan, Laboratoire des Sciences du Climat et de l'Environnement, Commissariat à l'Energie Atomique, 91191 Gif sur Yvette, France. (fmbreon@cea.fr)

I. Grant, CSIRO Atmospheric Research, Aspendale, VIC 3195, Australia. M. Leroy, Medias-France, CNES, 31055 Toulouse, France. 